\title{
Uso potencial de resíduo de uvas no desenvolvimento de produtos funcionais: uma
}

\section{revisão}

\author{
Potential use of grape residue in the development of functional products: a review \\ Uso potencial de residuos de uva en el desarrollo de productos funcionales: una revisión
}

Recebido: 18/12/2021 | Revisado: 26/12/2021 | Aceito: 29/12/2021 | Publicado: 30/12/2021

\author{
Claudia Nepomuceno da Silva \\ ORCID: https://orcid.org/0000-0002-2679-0640 \\ Universidade de Pernambuco, Brasil \\ E-mail: claudia.nsilva@upe.br \\ Daiane Nepomuceno da Silva \\ ORCID: https://orcid.org/0000-0002-0218-2851 \\ Universidade de Pernambuco, Brasil \\ E-mail: daiane.nsilva2@upe.br \\ Cedenir Pereira de Quadros \\ ORCID: https://orcid.org/0000-0002-5989-2248 \\ Universidade Federal do Vale do São Francisco, Brasil \\ E-mail: cedenir.quadros@univasf.edu.br \\ Claudileide de Sá Silva \\ ORCID: https://orcid.org/0000-0002-3677-3000 \\ Universidade de Pernambuco, Brasil \\ E-mail: Claudileide.silva@upe.br
}

\begin{abstract}
Resumo
O desperdício de alimentos é preocupante, pois pelo menos 1,4 bilhão de toneladas de alimentos são desperdiçados no mundo anualmente. Diversos aspectos ambientais, sociais e econômicos contribuem para o aumento desses números, todavia metade do desperdício de alimentos no mundo poderia ser evitado por medidas preventivas. Desse modo, o presente trabalho teve como objetivo avaliar através de revisão da literatura, o potencial uso de subprodutos da uva, como por exemplo o bagaço, no desenvolvimento de produtos funcionais. Para tanto, foram utilizados os descritores "uva", "uva qualidade inferior", "uva exportação", "uva alimento funcional", "alimentos funcionais bagaço de uva", "casca de uva alimento funcional", "uva de refugo", "grape", "low quality grape", "export grape", "functional food grape", "functional food grape pomace", "grape skin functional food", "junk grape". Como base de dados foram utilizadas a PubMed (National Library of Medicine), Portal de Periódicos Capes, LILACS (Literatura latinoamericana e do caribe em ciências da saúde), Science Direct, Scorpus e Crossref, utilizando descritores da língua inglesa e portuguesa, e selecionando os artigos publicados entre os anos de 2000 a 2022 . Foram encontrados 942 artigos $($ PubMed= 546 ScienceDirect= 373; Scielo= 25), dos quais 80 artigos foram selecionados, e desses 68 foram excluídos por não se enquadrarem nos critérios de inclusão que eram, produtos funcionais desenvolvidos através dos subprodutos da uva que seriam descartados, e tendo, como também ano de publicação do estudo ter ocorrido a partir do ano de 2000. E assim foram utilizados 15 artigos. Os trabalhos em sua maioria aplicaram a uva para o desenvolvimento de farinhas para a produção de bolos e o enriquecimento de iogurtes, além de produzirem cookies, geleias e barras de cereais. Através da revisão realizada foi possível evidenciar que a utilização de um resíduo vitivinícola que tem potencial para conferir benefícios à saúde através do desenvolvimento de produto funcionais, reduzindo a quantidade destes resíduos na indústria e no meio ambiente, com possibilidade também de reduzir custos na produção dos alimentos.
\end{abstract}

Palavras-chave: Alimentos funcionais; Fibras; Antioxidantes; Vitis Labrusca L; Subproduto.

\begin{abstract}
Food waste is of concern, as at least 1.4 billion tons of food are wasted worldwide each year. Several environmental, social and economic aspects contribute to the increase in these numbers, however half of the food waste in the world could be avoided by preventive measures. Thus, this study aimed to evaluate, through a literature review, the potential use of grape by-products, such as bagasse, in the development of functional products. For this purpose, the descriptors "grape", "low quality grape", "export grape", "grape functional food" were used, "functional food grape pomace", "grape skin functional food", "junk grape", "grape", "low quality grape", "export grape", "functional food grape", "functional food grape pomace", "grape skin functional food", "junk grape". The database used was PubMed (National Library of Medicine), Capes Journal Portal, LILACS (Latin American and Caribbean Literature in Health Sciences), Science Direct, Scorpus and Crossref, using descriptors in English and Portuguese, and selecting articles published between the years 2000 to 2022. 942 articles were found $($ PubMed= 546 ScienceDirect $=373 ;$ Scielo= 25),
\end{abstract}


of which 80 articles were selected, and of these 68 were excluded for not meeting the inclusion criteria that they were functional products developed through the by-products of the grape that would be discarded, and having, as well as the year of publication of the study, occurred from the year 2000. And so, 15 articles were used. Most of the works applied the grape to the development of flour for the production of cakes and the enrichment of yogurts, in addition to producing cookies, jellies and cereal bars. Through the review carried out, it was possible to show that the use of a wine residue that has the potential to provide health benefits through the development of functional products, reducing the amount of these residues in industry and the environment, with the possibility also of reducing costs in the production of foods.

Keywords: Functional foods; Fibers; Antioxidants; Vitis Labrusca L; By-product.

\section{Resumen}

El desperdicio de alimentos es motivo de preocupación, ya que cada año se desperdician al menos 1.400 millones de toneladas de alimentos en todo el mundo. Varios aspectos ambientales, sociales y económicos contribuyen al aumento de estas cifras, sin embargo, la mitad del desperdicio de alimentos en el mundo podría evitarse con medidas preventivas. Así, este estudio tuvo como objetivo evaluar, a través de una revisión de la literatura, el uso potencial de subproductos de la uva, como el bagazo, en el desarrollo de productos funcionales. A tal efecto, se utilizaron los descriptores "uva", "uva de baja calidad", "uva de exportación", "alimento funcional de uva", "alimento funcional orujo de uva", "alimento funcional piel de uva", "uva chatarra", "uva "," uva de baja calidad "," uva de exportación "," uva alimenticia funcional "," orujo de uva alimenticio funcional "," alimento funcional piel de uva "," uva basura ". La base de datos utilizada fue PubMed (Biblioteca Nacional de Medicina), Capes Journal Portal, LILACS (Literatura Latinoamericana y del Caribe en Ciencias de la Salud), Science Direct, Scorpus y Crossref, utilizando descriptores en inglés y portugués, y seleccionando artículos publicados entre los años 2000 al 2022. Se encontraron 942 artículos $($ PubMed $=546$ ScienceDirect $=373$; Scielo $=25)$, de los cuales se seleccionaron 80 artículos, y de estos 68 fueron excluidos por no cumplir con los criterios de inclusión de ser productos funcionales desarrollados a través de los subproductos de la uva que se descartaría, y que, además del año de publicación del estudio, se da desde el año 2000. Así, se utilizaron 15 artículos. La mayoría de los trabajos aplicaron la uva a la elaboración de harinas para la elaboración de tortas y enriquecimiento de yogures, además de la elaboración de galletas, gelatinas y barritas de cereales. A través de la revisión realizada, se pudo evidenciar que el uso de un residuo de vino que tiene el potencial de brindar beneficios para la salud a través del desarrollo de productos funcionales, reduciendo la cantidad de estos residuos en la industria y el medio ambiente, con la posibilidad también de reducción de costos en la producción de alimentos.

Palabras clave: Alimentos funcionales; Fibras; Antioxidantes; Vitis Labrusca L; Subproducto.

\section{Introdução}

A fruticultura é um dos segmentos mais importantes, respondendo por $25 \%$ do valor da produção agrícola nacional, porém um grande problema enfrentado pelos fruticultores é a conservação dos frutos maduros (De Souza Barbosa et al., 2014). A falta de tecnologias adequadas para o melhor aproveitamento dos frutos tem resultado em um alto índice de desperdício (Machado et al., 2015).

O desperdício de alimentos é uma problemática que preocupa as autoridades, visto que, segundo dados de 2014 da Organização das Nações Unidas para a Alimentação e Agricultura, 1,4 bilhão de toneladas de alimentos são desperdiçados no mundo anualmente (Fao, 2014).

O Brasil é o terceiro maior produtor mundial de frutas, com uma produção que supera 44 milhões de toneladas em 2014 (Abrasfrutas, 2020). A base agrícola da cadeia produtiva das frutas abrange mais de 2,2 milhões de hectares, gera mais de 4 milhões de empregos diretos e indiretos, além disso, para cada 10.000 dólares investidos em fruticultura, são gerados 3 empregos diretos permanentes e dois empregos indiretos (Machado, 2015).

Neste contexto, a região Nordeste do Brasil representa a segunda maior região produtora de uvas do país com aproximadamente $32 \%$ da produção nacional, tendo sua produção voltada tanto para uvas de mesa quanto uvas para processamento de sucos e vinhos (De Mello, 2018). Atrás apenas da região Sul que detém aproximadamente 52\%, mas que se destinam em sua maioria à produção de sucos e vinhos (Mello, 2017). 
O estado de Pernambuco produziu em 2017 cerca de 390,3 mil toneladas de uvas, superando a produção do estado da Bahia, que nos anos de 2005 a 2007 ultrapassou 100 mil toneladas de produção de uvas, e no ano de 2017 produziu cerca de 51,09 mil toneladas em uma área de 2.229 ha (Mello, 2017; De Souza Leão et. al., 2016).

É importante ressaltar que a uva é composta de açúcares, ácidos, pectina, gomas, compostos aromáticos e compostos fenólicos. Ela é considerada uma das maiores fontes de compostos fenólicos quando comparada a outras frutas e vegetais, possuindo assim vários efeitos benéficos à saúde (Perin et. al., 2011).

No entanto, o não seguimento aos parâmetros de qualidade, os quais são determinados pelos importadores, provoca o desperdício dos frutos não adequado a estes. Dentre os padrões, estão: coloração, calibre (mm), acidez, tamanho de cacho, quantidade de cachos dentro da cumbuca, peso da cumbuca, presença de corpos estranhos (insetos, por exemplo) e de bagas podres, organização das cumbucas na caixa, tipo de fechamento do bolsão e selagem (Araújo Neto, 2019).

Segundo Araújo, (2019) as bagas que sofreram desgastes durante o transporte são colocadas em embalagens e depositadas em contentores e destinadas à sala de refugo, a qual fica localizada dentro do perímetro do "Packing House". Nesse local as bagas aguardam o momento que são recolhidas por pequenos produtores para serem comercializadas.

A indústria tem como um desafio a transformação destes resíduos em subprodutos de valor agregado, destinando-os ao reprocessamento, visando reduzir impactos negativos causados (Caldas et al., 2018). Estima-se que 40\% das frutas são perdidas entre a colheita no campo até a chegada ao mercado consumidor final. Essas perdas podem ser de natureza quantitativa ou qualitativa, ocasionando assim redução no seu valor comercial (Machado et al., 2015; Almeida et al., 2019).

Apesar de o Brasil se caracterizar como um país altamente produtor, é também um dos países onde mais se perdem alimentos durante essa etapa. A falta de conhecimento dos processos fisiológicos dos frutos, bem como a falta de infraestrutura adequada e de uma logística de distribuição são os principais fatores responsáveis pelo elevado nível de perdas pós-colheita observadas no país (Ferreira, 2017).

Por outro lado, o resíduo proveniente da produção de vinhos e sucos também é preocupante, tendo em vista que a indústria de vinho gerou em 2013 quase 3,8 milhões de toneladas de bagaço de uva (BU), causando problema ambiental, social e econômico (Caldas et. al., 2018). Com isso, o destinamento final dos resíduos da indústria vinícola enfrenta um grande desafio para transformar estes em subprodutos, destinando-os ao reprocessamento para redução do impacto ambiental (Caldas et. al., 2018).

E diante do exposto, acredita-se que a utilização de frutas de qualidade inferior e subprodutos, como o bagaço proveniente da indústria vinícola e de sucos, podem ser utilizados no processamento para desenvolvimento novos produtos com características funcionais.

Desse modo, o presente trabalho tem por objetivo avaliar o uso potencial de resíduo de uvas no desenvolvimento de produtos através de uma revisão da literatura.

\section{Metodologia}

Realizou-se uma revisão sistemática da literatura científica sobre a utilização do resíduo de uva para produção de produtos funcionais. Para a seleção dos artigos utilizou-se as bases de dados Pubmed (National Library of Medicine), Portal de Periódicos Capes, LILACS (Literatura latino-americana e do caribe em ciências da saúde), Science Direct, Scorpus e Crossref, utilizando descritores da língua inglesa e portuguesa, e selecionando os artigos publicados entre os anos de 2000 a 2022.

Para a pesquisa em língua portuguesa foram usados os descritores uva", "uva qualidade inferior", "uva exportação", "uva alimento funcional", "alimentos funcionais bagaço de uva", "casca de uva alimento funcional", "uva de refugo"; e na língua inglesa foram utilizados os termos "grape", "low quality grape", "export grape", "functional food grape", "functional food grape pomace", "grape skin functional food", "junk grape". 
Os critérios de inclusão foram: produtos funcionais desenvolvidos através dos subprodutos da uva que seriam descartados, e tendo, como também ano de publicação do estudo ter ocorrido a partir do ano de 2000. Os critérios de exclusão foram: estudos que não desenvolveram produtos funcionais e/ou com ano de publicação anterior ao ano de 2000.

O presente estudo não requer autorização do Comitê de Ética em Pesquisa tendo em vista que se trata de uma revisão da literatura, de acordo com a Resolução do Conselho Nacional de Saúde (CNS) n 466, 12 de dezembro de 2012.

\section{Resultados}

Foram encontrados pelo menos 942 artigos (Pubmed= 546 ScienceDirect= 371; Scielo= 25), através da busca direta nestes sites. Inicialmente 104 artigos foram excluídos em virtude de duplicidade e 758 por não se tratarem de produtos alimentares desenvolvidos com o resíduo de uva. Sendo assim, foram pré-selecionados 80 artigos, dos quais 62 foram excluídos por não se enquadrarem nos critérios de inclusão (figura 1). Assim, nesta revisão foram utilizados 18 estudos.

Figura 1: Diagrama de fluxo desta revisão sistemática.
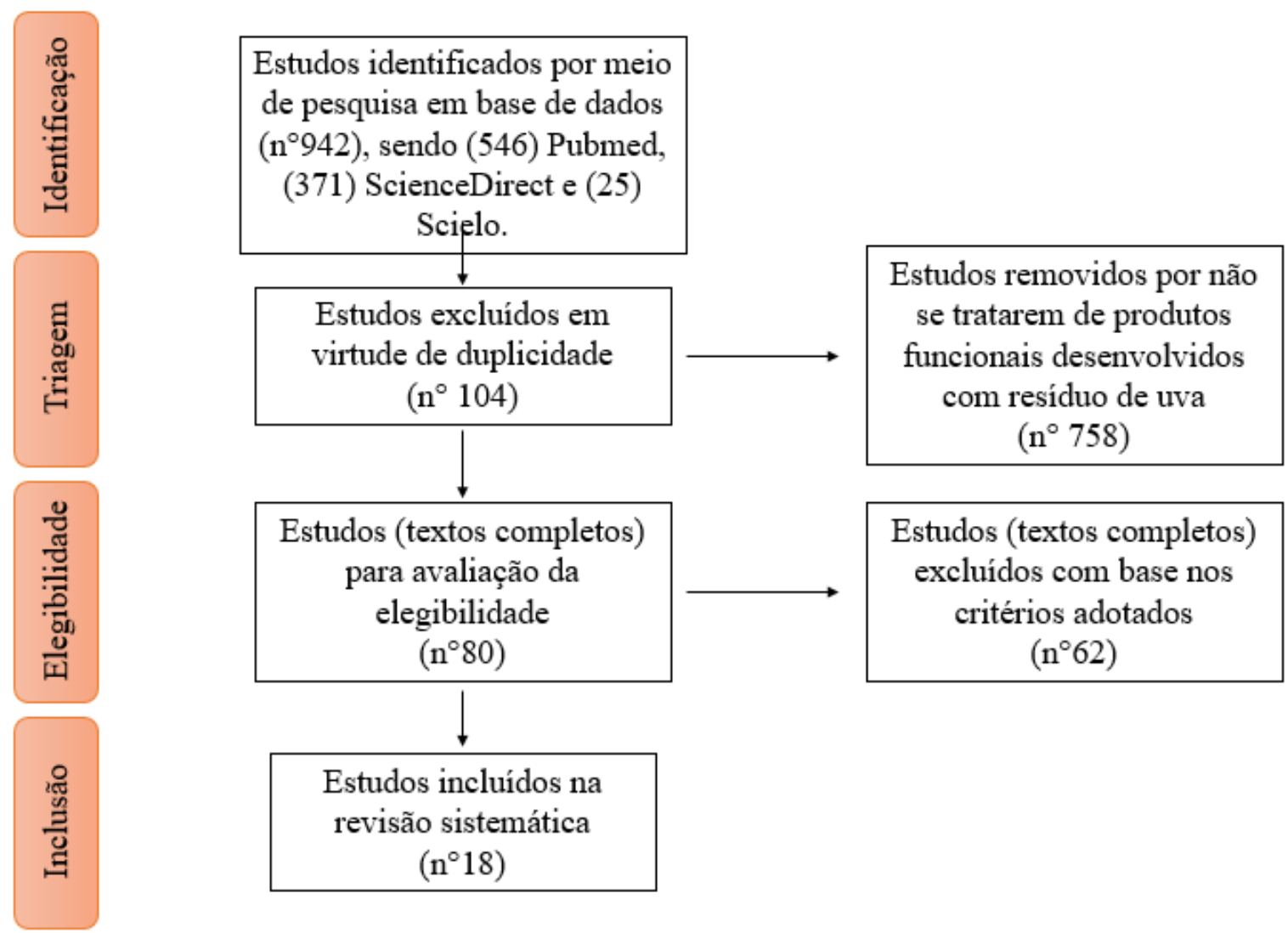

Fonte: Autores. 
Os dados referentes aos artigos incluídos nesta revisão são apresentados na tabela 1, onde são abordados resultados de caracterização geral dos estudos, como autores, ano de publicação, total de participantes, os tipos de produtos e os tipos de grupos de comparação.

Tabela 1. Delineamento amostral, objetivos e principais resultados encontrados por estudos desenvolvidos de 2000 a 2022 , Petrolina, 2021.

\begin{tabular}{cl}
\hline Autor (es) & \multicolumn{2}{c}{ Delineamento } \\
\hline NAKOV et & Foi analisada a composição química, \\
al., 2020 & propriedades nutricionais e características \\
& físicas de bolos preparados substituindo a \\
& farinha de trigo para pães por bagaço de uva \\
& em pó $4 \%, 6 \%, 8 \%$ e $10 \%$.
\end{tabular}

\section{HUERTA, \\ M.M. et al.}

2018.

SILVA, R.; QUADROS,

C.P.; SILVA C.S., 2020.

RAMOS, $R$. V. R. et al., 2020.

\section{Elaboração de bolos, utilizando três} tratamentos de pré-misturas com diferentes concentrações de farinha do bagaço de uva (FBU) $(15,30$ e $45 \%)$, além da pré-mistura controle $(0 \%)$, avaliadas sensorialmente.
BENDER, et al., 2016.
Elaboração de bolos com concentrações diferenciadas, sendo as amostras de $0 \%, 10 \%$, $20 \%$ e $30 \%$, as quais foram avaliadas sensorialmente por 50 provadores não treinados, de ambos os sexos, maiores de 18 anos.

Foi utilizado bagaço de uva da cultivar Marselan (Vitis vinifera) proveniente do processo de vinificação, e as amostras submetidas às análises de composição química (umidade, cinzas, proteína, lipídeos, fibra alimentar total e carboidratos, $\mathrm{pH}$, compostos fenólicos e cor).
Reaproveitamento do bagaço de uva a partir da produção de uma farinha sua avaliação e aplicação em pré-misturas para bolo.

Avaliação da influência do pó de bagaço de uva (Vitis vinífera), um subproduto do vinho na produção de bolos.
Elaboração de bolo enriquecido com fibras e antioxidante a partir da farinha do bagaço de uva Cabernet Sauvignon (Vitis vinifera L.)

Elaboração de snack com a farinha de casca de uva (FCU) e o efeito de sua inclusão nos parâmetros nutricionais, tecnológicos e sensoriais.

Elaboração de farinhas vegetais na sua forma integral ou de suas partes alimentícias não convencionais, e caracterização das farinhas obtidas, com vistas à alimentação humana.
A adição de quantidades crescentes de pó de bagaço de uva aumentou gradualmente as cinzas, lipídios, proteínas, fibras, compostos fenólicos livres, antocianinas e teor total de polifenóis, bem como capacidade antioxidante (DPPH, FRAP), enquanto diminuição da umidade e pH. Os principais fenólicos fornecidos pelo bagaço da uva foram catequina, ácido gálico, quercitina, ácido protocatecuico, caempferol e apigenina. O conteúdo de ácidos fenólicos e flavonoides aumentou de 4,1 mg / $\mathrm{kg}$ de MS (controle) para 26,4-60,9mg / kg de MS (bolo com bagaço de uva em pó $4 \%-10 \%)$. As coordenadas de cor $\mathrm{L}$ * e a $*$ diminuíram, enquanto $\mathrm{b} *$ aumentada. $\mathrm{O}$ bolo contendo $4 \%$ de bagaço de uva em pó apresentou a melhor qualidade sensorial.

Os maiores escores foram obtidos para prémistura com $45 \%$ de FBU para todos os atributos avaliados, e sendo selecionada para demais análises. No entanto, a pré-mistura selecionada (PMS) obteve maiores teores de cinzas, lipídeos e fibra alimentar total quando comparada a PMC, embora os elaborados a partir da PMS obtiveram menor volume e maior firmeza quando comparados com os bolos preparados a partir da PMC. Em todas as amostras houve estabilidade microbiológica, e as amostras enriquecidas com FBU apresentaram teores consideráveis e maiores do que a amostra controle ao avaliar o conteúdo de compostos bioativos e atividade antioxidante.

A preparação com $10 \%$ de farinha proveniente do bagaço de uva obteve $88 \%(n=44)$ de aceitação na avaliação global e maior índice de aceitação entre as amostras, revelando que $86 \%(n=43)$ certamente ou provavelmente comprariam o produto, sendo a mesma submetida às análises físico-químicas. Houve redução em $40 \%$ do teor de gorgura quando comparado ao bolo tradicional.

A FCU foi incluída na formulação de snacks extrusados nas concentrações de $9 \%$ e $18 \%$, em substituição à farinha de milho, representando $5 \%$ e $10 \%$ de fibra, respectivamente. Fibra $(58,01 \%)$, carboidratos $(17,62 \%)$ e cinzas $(12,46 \%)$ foram os principais constituintes da farinha de casca de uva, a qual teve $\mathrm{pH}$ de 3,51. Resveratrol $(6,14$ mg.g-1), luteolina $(5,16 \mathrm{mg} . \mathrm{g}-$ 1) e kaempferol (3,01 mg.g-1) foram os compostos fenólicos detectados em maior quantidade na FCU, indicando presença de antioxidantes na farinha. A formulação de snack contendo 9\% (5\% fibra) de FCU apresentou melhores resultados de aceitação com relação aos atributos cor, aroma e textura, comparada à formulação de snack padrão.

As farinhas apresentaram alto teor de fibras $(17,7$ a $48,94 \mathrm{~g} / 100 \mathrm{~g})$ e compostos fenólicos $(778,81$ a $2708,11 \mathrm{mg}$ EAG/100g), e baixo teor de lipídeos $(0,70$ a $3,40 \mathrm{~g} / 100 \mathrm{~g})$. Para a quantidade de proteínas, a farinha de berinjela foi a que mais se destacou $(13,81 \mathrm{~g} / 100 \mathrm{~g})$, podendo ser uma alternativa aos indivíduos que não consomem 
SIEBENEIC Foram elaborados pães com farinha de trigo HLER, $\boldsymbol{T}$. J. adicionada de concentrações de $0,1 \mathrm{~g} / \mathrm{g}$ e 0,2 et al., 2016. $\mathrm{g} / \mathrm{g}$ de farinha de uva, sendo avaliados o tamanho dos pães, sua massa, compostos fenólicos totais e compostos com atividade antioxidante dos produtos, além de testes de aceitação sensorial.

SILVA, F.A. Avaliação das características físico-químicas e (2020) dos compostos bioativos por meio da análise qualitativa e quantitativa dos compostos fenólicos totais, antocianinas totais e atividade antioxidante. Em seguida, os produtos foram incorporados em iogurtes caprinos e realizado o estudo do impacto em suas propriedades.
BENNEMAN Foram elaboradas cinco formulações $\boldsymbol{N}$, G. D. et de muffins, sendo: F1: padrão (0\%) e as demais al., 2016. adicionadas de $25 \%$ (F2) e $50 \%$ (F3) de FCU do cultivar Ancellotta; $25 \%$ (F4) e 50\% (F5) da FCU do cultivar bordô. Participaram da análise sensorial 50 provadores, não treinados, com idade acima de 18 anos.

VANDERLE

I, D. R.,

SILVA, C.S.; QUADROS,

C. P, 2020.

Foram elaboradas três formulações de geleia $0 \%$ (controle), $70 \%$ e $90 \%$ de concentração de bagaço de uva, as geleias foram avaliadas por 50 provadores não treinados, compostos por julgando os atributos: textura, aroma, sabor e avaliação global.
Objetivou verificar a aceitabilidade sensorial de muffins adicionados de farinha de casca de uva (FCU) das cultivares Ancellotta e Bordô, bem como determinar a atividade microbiológica destas farinhas.

Produção de geleia de chia e bagaço da uva.

Avaliar o efeito do tempo e da temperatura de secagem sobre o teor de compostos fenólicos totais e a capacidade antioxidante do bagaço de uva para posterior obtenção de uma farinha e sua utilização na formulação de barras de cereais. resíduo (SQR). Foram formulados quatro tipos de barra de cereais, variando a quantidade de farinha de bagaço de uva, nas quais foram utilizados os seguintes ingredientes: açúcar $(19 \%)$, glicose $(7 \%)$, gergelim $(2 \%)$, fibra de trigo $(9 \%)$, aveia $(2 \%)$, flocos de arroz $(10 \%)$, castanha do Pará (17\%), água (34\%), de acordo com Balestro et al. (2011). As barras formuladas tiveram diferentes concentrações de farinha de bagaço de uva $(22,2 \%, 55,6 \%$ e $100 \%)$ em substituição à fibra de trigo,

FAGNANI et A formulação foi composta por leite al., 2020. pasteurizado padronizado à $2 \%$ de gordura $89,87 \% \mathrm{~m} / \mathrm{m})$, oligossacarídeos - 6,0\% $\mathrm{m} / \mathrm{m}(9$ galacto-oligossacarídeo: $\quad 1 \quad$ frutooligossacarídeo, scGOS/lcFOS Danone Nutricia), creme de leite pasteurizado - $2 \%$ $\mathrm{m} / \mathrm{m}$ (Frimesa), leite em pó desnatado - 1,0\% $\mathrm{m} / \mathrm{m}$ (Frimesa), polidextrose - $0,6 \% \mathrm{~m} / \mathrm{m}$ (Litesse ${ }^{\circledR}$, Danisco), farinha de semente de uva orgânica $-0,4 \% \mathrm{~m} / \mathrm{m}$ (Econatura), edulcorante

Elaboração iogurte concentrado enriquecido com farinha de semente de uva, avaliando a velocidade de fermentação e as características físicas, químicas e microbiológicas.
Elaboração de pães com a incorporação de farinha de uva à farinha de trigo convencional e avaliar o efeito dessa substituição em características tecnológicas, sensoriais e nutricionais dos produtos.

Avaliação dos compostos fenólicos presentes no preparado de uva Isabel e na farinha de seus resíduos elaborados utilizando adoçantes naturais (agave e xilitol), bem como avaliação do impacto da incorporação desses produtos na qualidade nutricional, funcional e sensorial de iogurte caprino. produtos de origem animal.

Para os quesitos aroma, textura, sabor e aceitação global não houve diferença $(p>0,05)$ na aceitação das formulações elaboradas. Assim, a adição de farinha de uva à formulação de pães não alterou as características tecnológicas dos produtos, e aumentou suas propriedades antioxidantes. Quanto à análise sensorial, observou-se que os produtos com farinha de uva apresentam aceitação semelhante ao produto sem a sua incorporação.

A uva e seus produtos apresentaram teores relevantes de açúcares e ácidos orgânicos em sua composição, destacando-se na farinha o considerável teor de fibras $(20,14-21,95 \mathrm{~g} / 100$ g). Os iogurtes caprinos elaborados com adição de $L$. acidophilus e produtos de uva Isabel mostraram-se ricos em compostos fenólicos e com elevada atividade antioxidante. Também apresentaram contagens adequadas do probiótico L. acidophilus $(7,96-8,63 \mathrm{log} \mathrm{UFC} / \mathrm{mL})$ durante todo o período de armazenamento, com a formulação incorporada do preparado e farinha exibindo as maiores contagens ao fim dos 28 dias. A adição do preparado e da farinha de uva Isabel aumentou a aceitação desse iogurte pelos consumidores.

A partir da análise sensorial, percebeu-se que as maiores notas para F1 e F2 foram verificadas nos atributos aparência, cor e aceitação global. Porém, destaca-se que as amostras F2 e F4 foram aquelas com maior aceitabilidade, comparadas à F3 e F5. Em relação à atividade microbiológica, não foram encontrados valores significativos, assegurando os critérios de controle de qualidade das amostras. Portanto, a adição de até $25 \%$ de FCU de ambos as cultivares foi bem aceito pelos provadores.

Os resultados obtidos da análise sensorial, revelaram que a amostra de geleia contendo $70 \%$ de bagaço obteve boa aceitação. Esta amostra obteve para os atributos textura, sabor aroma e impressão global $48 \% \quad(n=24), 50 \% \quad(n=25)$ e $56 \%(\mathrm{n}=28)$ para gostei muito, respectivamente, já intenção de compra de $16 \%(\mathrm{n}=8)$ para certamente compraria e $60 \% \quad(\mathrm{n}=30)$ provavelmente compraria. As características sensoriais avaliadas foram bem aceitas pelos entrevistados.

Os tratamentos apresentaram discrepância significativa quanto à retenção dos compostos fenólicos totais e capacidade antioxidante. O bagaço seco a $60^{\circ} \mathrm{C}$ apresentou os maiores valores para fenólicos totais e capacidade antioxidante, sugerindo que esta temperatura foi capaz de inativar enzimas que catalisam a degradação dos compostos fenólicos. Foram realizados novos testes de secagem e a partir destes, foram obtidas a farinha do bagaço de uva que, após ser caracterizado, foi utilizado como ingrediente em diferentes concentrações para a formulação de barras de cereais. Esses produtos foram avaliados frente ao conteúdo de compostos bioativos e capacidade antioxidante. Aquelas com os maiores teores de farinha apresentaram as maiores concentrações de compostos bioativos e capacidade antioxidante.
O tempo de fermentação foi de $8 \mathrm{~h}$, alcançando pH 4,5 e contagens de bactérias ácido láticas de 9 $\log \mathrm{UFC} / \mathrm{mL}$. Após 24h, as contagens de coliformes totais, bolores e leveduras porcentagem proteica e lipídica atenderam aos para iogurtes parcialmente desnatados. A textura e a viscosidade mostraram boa firmeza do coágulo e ficaram dentro dos valores esperados para iogurtes concentrados. A capacidade parâmetros exigidos pela legislação brasileira 
- 0,03\% m/m (Doremix Sweet CSS, Doremus) e cultura láctea - $0,1 \% \mathrm{~m} / \mathrm{m}$ (YO-MIX® 499 LYO 100 DCU, Danisco).

POIANI, et. Foram utilizadas cinco formulações distintas, al., 2019. em diferentes temperaturas e tempos de assamento.

TONON, G. Foram elaboradas 3 formulações diferentes, C. (2019) que substituíram a farinha de trigo integral por farinha de uva nas porcentagens de $15,10 \mathrm{e}$ $5 \%$. Foi aplicada análise sensorial com 71 provadores não treinados a fim de verificar a aceitabilidade das amostras utilizando escala hedônica e sua intenção de compra. Os dados foram tratados através da análise de variância (ANOVA) e a comparação de médias foi feita utilizando teste de Tukey com $5 \%$ de significância

ABREU, J. Foi realizada análises físico-químicas na P.de. 2018. farinha de casca de uva orgânica, o Dissertação planejamento fatorial $2^{3}$ foi utilizado para o de Mestrado. desenvolvimento do cookie com variáveis independentes farinha de casca de uva orgânica, gordura interesterificada e sacarose. A análise sensorial de aceitação foi utilizada para avaliar as variáveis dependentes, aparência, aroma, sabor, textura e impressão global.

DE MELO, Os cookies foram avaliados quando à V.S.et al., composição centesimal e aceitabilidade por 2010. provadores não treinados e aleatórios $(n=90)$.

PERIN, E. O estudo foi realizado por meio de análises C., \& Schott, físico-químicas da farinha, sendo elas $\mathrm{pH}$, I. B., 2011. acidez, umidade e cinzas para caracterizá-las. A partir da farinha, elaborou-se formulações de cookies com substituições de 5, 10 e $15 \%$ da farinha de trigo pela farinha de bagaço. Posteriormente foi realizada análise sensorial com 80 julgadores sendo aplicado um teste de aceitação e intenção de consumo, a fim de determinar a amostra mais aceita para realização posterior dos compostos bioativos.

DE ASSIS, Foram analisados sorvetes em concentrações C. F. et al, de 15,20 e $25 \%$ de farinha de bagaço de uva. 2018. As amostras foram submetidas ao teste de aceitação e intenção de compra, avaliadas por 50 provadores não treinados. Esses dados foram submetidos à análise de variância e ao o teste de Tukey, utilizando o nível de 5\% de significância.
Desenvolvimento de cookies com mix de farinhas (uva, linhaça dourada, amido de milho) e avaliar suas características físicas, tecnológicas e sensoriais.

Elaboração de cookies de farinha de trigo integral adicionados de farinha de uva e óleo de semente de uva, e realização de avaliação de sensorial de cookies feitos ambos obtidos do bagaço de uva da indústria de sucos e vinhos.

Desenvolvimento de biscoito tipo cookie com características funcionais através da adição de farinha de casca de uva orgânica.

Foi desenvolvido uma formulação de cookie, substituindo-se $25 \%$ da farinha de trigo por farinha de semente de uva.
Elaboração de uma farinha a partir do bagaço gerado na produção de suco de uva, para desenvolver um biscoito adicionado desta farinha e avaliar os compostos bioativos presentes no resíduo, na farinha e no biscoito produzido.

Foi desenvolvido um tipo de sorvete com adição de farinha obtida do bagaço de uva em substituição total a corantes e essências com o objetivo de avaliar a sua aceitabilidade e a composição nutricional da farinha.

Foi observado um alto teor de fibras e grande quantidade de antioxidantes, observado pelos métodos DPPH, ABTS, FRAP e ORAC, além de um elevado conteúdo de compostos fenólicos totais, tendo como principal composto, detectado na análise de HPLC, o resveratrol. O emprego de farinha de casca de uva orgânica mostrou-se viável no desenvolvimento do cookie apresentando boa aceitação.

Os elaborados com farinha de semente de uva evidenciaram, em 100g, cerca de 447,18 calorias, $63 \%$ de carboidratos, $5 \%$ de proteína, $19 \%$ de lipídios e $5,7 \%$ de fibras. Na aceitação global, o cookie de uva apresentou média de 8,03, correspondendo a "gostei muito" na escala hedônica.

O cookie com adição de $10 \%$ de farinha de bagaço de uva foi o que teve maior aceitação, sendo avaliado os polifenóis totais, antocianinas totais e capacidade antioxidante do mesmo. A farinha apresentou-se dentro dos limites estipulados pela legislação quanto as variáveis físico-químicas analisadas. Com relação a análise de compostos bioativos o bagaço, a farinha e o cookie, apresentaram os compostos estudados, sendo encontrados em maior quantidade no bagaço.

Os resultados demonstraram que o sorvete com $15 \%$ de farinha de bagaço de uva em sua composição apresentou maior aceitação, entretanto, a formulação com $25 \%$ da farinh apresentou valores maiores quanto à intenção de compra. A farinha de bagaço de uva apresentou alto teor de fibras $(21,60 \mathrm{~g} / 100 \mathrm{~g})$, carboidratos e proteínas, e baixo teor de lipídeos e está de acordo com os critérios propostos pela legislação da ANVISA. O produto pode ser considerado promissor para a indústria de alimentos, apresentando características nutricionais desejáveis com baixo custo.

Coeficiente de consistência $(\mathrm{K})$ e os valores do índice de comportamento do fluxo de CS as amostras mudaram entre 8,10 e $28,50 \mathrm{~Pa}$.sn e $0,52-0,71$, respectivamente. Além disso, os parâmetros de firmeza e espalhabilidade foram determinados entre 0,80-1,42 $\mathrm{N}$ e 2,15-4,24 $\mathrm{N}$ $\mathrm{mm}$, respectivamente. Fenólico total o conteúdo em amostras de CS variou de 3415 a $13.754 \mathrm{mg}$ 
usando o projeto de método de mistura. As concentrações de açúcar, MOP e DGP mudaram entre 35 e 50, 0,066-8.000 e 0,23-15 $\mathrm{g} / 100 \mathrm{~g}$, respectivamente.
GAE / $\mathrm{kg}$ e sua digestibilidade variou entre $25 \%$ e $84 \%$. Além disso, a digestibilidade do resveratrol foi determinada entre $47,6 \%$ e $95,7 \%$ em diferentes concentrações de DGP. Todos os modelos estabelecidos descreveram com sucesso a relação com valores de $\mathrm{R} 2$ superiores a 0,800 .

Fonte: Autores.

\section{Discussão}

Os alimentos elaborados com os subprodutos de uvas, a depender da sua concentração nestes constituintes podem ser classificados como funcionais. Neste sentido, os "Alimentos funcionais são alimentos enriquecidos com nutrientes ou outras substâncias que fornecem benefícios à saúde além do seu valor nutricional” (Williamson, 2009).

Segundo a Legislação Brasileira, o alimento ou ingrediente que alegar propriedades funcionais deve, "além de funções nutricionais básicas, quando se tratar de nutriente, produzir efeitos metabólicos e/ou fisiológicos e/ou efeitos benéficos à saúde, devendo ser seguro para consumo sem supervisão médica” (Brasil, 1999).

O conceito de alimento funcional também se estende ao desenvolvimento de alimentos que beneficamente influenciam a microbiota intestinal através da incorporação de micro-organismos probióticos e/ou fibras prebióticas (Siró, 2008; Sangwan et al., 2011). A Associação Dietética Americana define alimentos com propriedades funcionais como alimentos com potencial de saúde ou que tem ingredientes que podem prover benefícios a saúde, além de conter os nutrientes tradicionais (Berry, 2002; Henry, 2010).

As fibras também são consideradas funcionais, e são compostos resistentes à ação das enzimas digestivas humanas, sendo constituídas de polissacarídeos não-amido, lignina (polímero de fenilpropano), carboidratos análogos (amido resistente e maltodextrina resistente), oligossacarídeos (com três ou mais unidades monoméricas) e alguns componentes de origem animal (quitina, quitosana, condroitina e colágeno (Machado, 2018). Alguns outros constituintes podem estar agregados à fibra alimentar, como os compostos fenólicos, fitatos, oxalatos, proteína de parede celular, cutina, suberina e ceras (Tungland e Mayer, 2002; Anderson et al., 2009; Howlett et al., 2010).

As fibras alimentares possuem propriedades físico-químicas, como a capacidade de retenção de água, viscosidade, fermentação, adsorção e ligação, volume, entre outras (Machado, 2018). Conforme o tipo de fibra ingerida, pode-se ter diferentes funções no organismo, como por exemplo, redução dos níveis de colesterol no sangue, aumento do bolo fecal, aumento da saciedade e ação como prebióticos (Gray, 2006; Buttriss \& Stokes, 2008).

Segundo a World Health Organization/Food and Agriculture Organization of the United Nations (Who/Fao, 2003), o consumo de fibras de fontes como frutas e vegetais está associado à redução do risco de desenvolvimento da obesidade e diabetes, doenças cardiovasculares e alguns tipos de câncer (cavidade oral, esofágico, gástrico e coloretal).

Segundo Tarcea et al. (2017), diversos estudos têm demonstrado que o consumo de fibras pelos consumidores tem sido insuficiente quando comparado aos valores estipulados para as recomendações diárias. Segundo a Resolução RDC n. 360/2003 da ANVISA (Agência Nacional de Vigilância Sanitária), os Valores Diários de Referência de nutrientes (VDR) para fibra alimentar é de 25 gramas. Na mesma resolução é apresentada a quantidade necessária para alegação de propriedade funcional e/ou de saúde no produto pronto para consumo, que no caso das fibras é no mínimo 2,5 g de fibras/porção do produto (Brasil, 2003).

Diversos outros países também regulamentam a quantidade mínima diária de consumo de fibras, como por exemplo os Estados Unidos, onde o FDA (Food and Drug Administration), recomenda a ingestão de 25 a 35 g de fibras por dia. Neste contexto, a adição de fibras em produtos industrializados é uma alternativa para o consumidor poder atingir o consumo mínimo diário necessário para obtenção dos vários benefícios à saúde atribuído às fibras (Giuntine et al., 2003; Saad, 2006). 
Neste contexto, a uva é composta basicamente de açúcares, ácidos, pectinas, gomas, compostos aromáticos e compostos fenólicos (Zopellaro et. al., 2019). Durante a maturação há uma evolução de alguns destes constituintes, dentre ele s: açúcares, ácidos, compostos fenólicos, vitaminas, minerais, ocorrendo então acumulação de açúcares, formação de taninos, diminuição de ácidos, formação de aroma, assim como o crescimento da baga da uva, cujo a sua composição química é influenciada pelo estágio de maturação, potencial genético, clima e manejo. (Perin et al, 2011).

Segundo Barcia (2014), as cascas de uvas apresentam teor de antocianinas iguais ou até maiores que frutas vermelhas como a amora e o mirtilo. Além da capacidade antioxidante, os flavonoides ainda possuem ações anti-inflamatória, antiplaquetária e antialérgica. Vários efeitos benéficos à saúde têm sido atribuídos aos compostos fenólicos presentes nas frutas, vegetais, chás e vinhos (Abe, et al., 2007). As uvas são consideradas uma das maiores fontes de compostos fenólicos quando comparadas a outras frutas e vegetais (Souza et. al., 2012).

Os antioxidantes estão presentes na casca e nas sementes das uvas de cor escura e podem agir sobre os radicais livres transformando-o em outro menos reativo, este pode ser chamado de "scavenger" ou aquele que completamente neutraliza o radical livre através da absorção de toda a energia de excitação, logo, este pode ser denominado "quencher" (Perin, 2011).

Há um interesse crescente em antioxidantes naturalmente encontrados em frutos para uso em fitoterápicos, a fim de substituí-los pelos antioxidantes sintéticos, os quais têm uso restrito devido a seus efeitos colaterais, tais como carcinogenicidade (Vedana, et al., 2009). Além disso, os antioxidantes naturais possuem a capacidade de melhorar a qualidade e a estabilidade dos alimentos, agirem como nutracêuticos e proporcionar, ainda, benefícios adicionais à saúde dos consumidores (Vedana, 2008).

Os compostos fenólicos também estão presentes em cascas e sementes das uvas e são estruturas químicas que apresentam hidroxilas e anéis aromáticos, nas formas simples ou de polímeros, que os confere o poder antioxidante (Silva, et al., 2018). Quando presentes em vegetais podem estar em formas livres ou complexadas a açúcares e proteínas (Machado, 2018).

Dentre eles, destacam-se os flavonoides, os ácidos fenólicos, os taninos e os tocoferóis como os antioxidantes fenólicos mais comuns de fonte natural (Jorge \& Ângelo, 2007).

Pertencente ao grupo de fitoquímicos e derivados da fenilalanina e tirosina, os compostos fenólicos podem ser classificados em flavonoides e não-flavonoides. Em uvas, pertencem ao primeiro grupo as antocianinas, principais responsáveis por sua coloração, os flavonóis (catequina, epicatequina e epigalocatequina), encontrados principalmente em sementes de uva e os flavonóis (caempferol, quercetina e miricetina), que atuam como copigmentos junto às antocianinas (Storck, C. R. et al, 2013). No segundo grupo estão os ácidos fenólicos, hidroxibenzóicos e hidroxicinâmicos. Além destes compostos, pode-se encontrar também o resveratrol, polifenol pertencente à classe dos estilbenos (Abe et al., 2007).

Além de suas características sensoriais peculiares, o grande interesse no consumo de uvas também se deve à presença dos compostos fenólicos relacionados a atividades benéficas à saúde humana, como antioxidante, cardioprotetor, anticarcinogênica, anti-inflamatório, antienvelhecimento e antimicrobiano (Xia et al., 2010). Os principais compostos fenólicos encontrados nas uvas são a catequina, os flavonoides e o resveratrol, cujas estruturas encontram-se apresentadas na Figura 5. 
Figura 5: Estrutura química de compostos fenólicos presentes em uvas. Fonte: adaptado de XIA et. al, 2010.

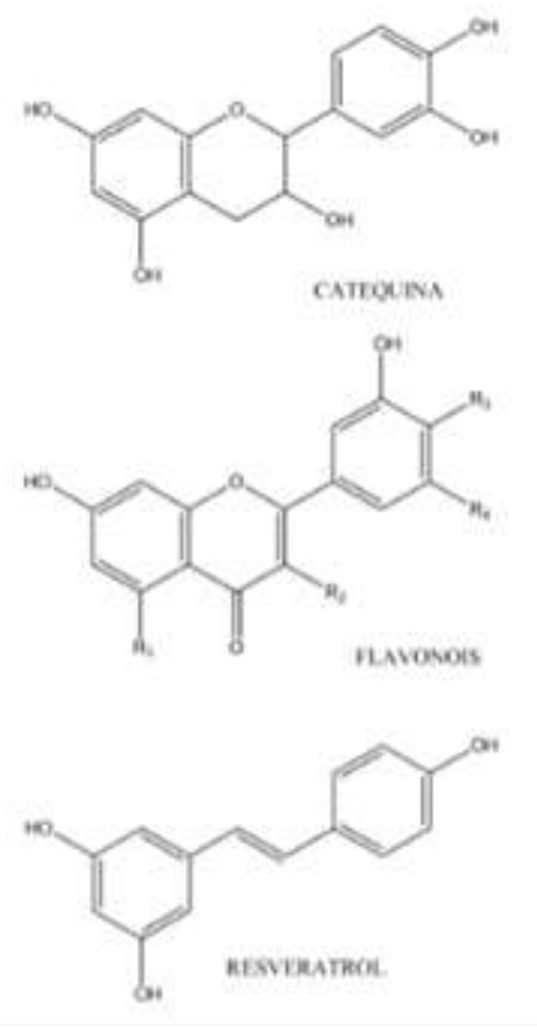

Fonte: Autores.

Segundo Zhao (2013), a realização de estudos vem buscando determinar as concentrações destes compostos nos alimentos mais consumidos e, em especial, nas frutas e em seus subprodutos. Pesquisas têm demonstrado o efeito protetor de dietas ricas em frutas e hortaliças contra doenças cardiovasculares e certos tipos de câncer, devido, em parte, aos antioxidantes contidos nestes alimentos (Rufino et al., 2007; Barreto, 2011; Costa, 2012; Tseng \& Zhao, 2013; Silva et al., 2014).

Os compostos fenólicos são os antioxidantes mais ingeridos na dieta humana, perfazendo um total de $1 \mathrm{~g} / \mathrm{dia}$, especialmente naquelas populações em que o consumo de alimentos de origem vegetal é maior (Silva, 2018).

O suco de uva e seus resíduos agroindustriais constituem uma importante fonte de compostos fenólicos, representados principalmente pelos flavonóis (catequinas e epicatequinas), antocianinas, resveratrol, quercetina e o kaempferol. A riqueza de polifenóis identificada nos sucos tem despertado o interesse crescente da população, o que reflete no consumo ascendente deste produto (Dani et al., 2007; Souza, 2008).

O teor de compostos fenólicos presentes nos produtos elaborados com uva também é fortemente influenciado por diversos fatores, como espécie, variedade da uva, maturidade, condições climáticas e métodos de elaboração dos produtos (Pinheiro, 2008). A concentração de fenólicos em sucos de uva, por exemplo, pode ser significativamente maior se durante sua fabricação as uvas forem esmagadas com o engaço, sementes e cascas, em comparação com processos que utilizam apenas a polpa da fruta (Silva, 2018). Assim as características nutricionais e funcionais apresentadas pelos seus resíduos estimulam o desenvolvimento de estratégias que viabilizem a sua inserção na alimentação humana, visto os diversos efeitos positivos sobre a saúde que poderiam advir do seu consumo regular (Natividade, 2010). 
O interesse neste tipo de resíduo para a extração de compostos de valor para a indústria de alimentos tem aumentado nos últimos anos, razão da importância de dimensionar a disponibilidade de bagaços de uvas provenientes da agroindústria de sucos e de vinho (Mello; Silva, 2014).

\subsection{Produtos elaborados com subprodutos de uva}

A exemplo do que ocorre com o resíduo da vinificação, sobretudo de uvas tintas, a utilização de uvas dos bagos de uva descartados por não atingirem o padrão para a exportação, podem agregar ganhos nutricionais se adicionados em produtos industrializados como cereal matinais, biscoitos, iogurte, geleias e doces (Barcia, 2014).

Estes subprodutos (resíduos) apresentam valor nutricional, visto que na maioria das vezes são compostos por proteínas, lipídios, carboidratos, vitaminas, minerais, fibras e compostos fenólicos, como os taninos, resveratrol, ácidos fenólicos e antocianinas (Sousa et al., 2014; Caldas et al., 2018).

Os produtos de conveniência vêm aquecendo o mercado, como os cookies, bolachas e barras de cereal, destacando-se principalmente pela sua praticidade, atendendo à atual demanda dos consumidores, relacionado aos aspectos nutricionais $\mathrm{e}$ funcionais (Da Silveira, M. A. G. et al., 2020). Porém, a utilização da farinha de casca de uva tem sido objeto de estudo para elaboração de barras de cereais a partir dos resíduos da uva resultante da produção de vinhos tintos por alguns estudos (Machado, 2018).

Sua constituição é expressante em flavonóis (quempferol, quercetina e miricetina), antocianinas (cianidina, delfinidina, peonidina e malvidina glicosiladas), estilbenos (resveratrol) e ácidos fenólicos (ácido cafeoiltartárico e o ácido pcumaroiltartárico) (Koyama et al, 2007; Saiko et al, 2008; Scola, 2014). Com isso, as uvas/subprodutos podem ser excelente fonte de compostos bioativos os quais podem imprimir atividade funcional nos alimentos nos quais são inseridas.

Os produtos de maior frequência encontrado/estudados utilizando resíduos de uva na produção de novos alimentos, que promovem e agregam valor funcional aos mesmos, mostram o quão são ricos em fibras e antioxidantes e bem aceitos pela população. Diversos estudos têm explorados a aplicação do bagaço de uva para a elaboração de cookies. Em estudo realizado por Perin e Schott, (2011), ao elaborar uma farinha a partir do bagaço gerado na produção de suco de uva, para desenvolver um biscoito tipo cookies e avaliar os compostos bioativos presentes no resíduo, na farinha e no biscoito produzido. Observou-se que o cookie com adição de $10 \%$ de farinha de bagaço de uva foi o que teve maior aceitação. No estudo, a análise de compostos bioativos no bagaço, na farinha e no cookie, apresentou os compostos estudados, sendo encontrados em maior quantidade no bagaço. No estudo realizado por Poiani et al. (2019), onde foram desenvolvidos cookies com mix de farinhas (uva, linhaça dourada, amido de milho) com o intuito de avaliar suas características físicas, tecnológicas e sensoriais. Foram utilizadas cinco formulações distintas, em diferentes temperaturas e tempos de assamento. $\mathrm{O}$ pH apresentou variação entre 5,38 e 6,44 (pré-cocção) e de 5,31 a 6,33 (pós-cocção). Quanto aos parâmetros de cor, a luminosidade variou entre 48,39 e 55,65, enquanto o croma $a^{*}$ foi de 3,38 a 6,44 e o croma b* de 13,21 a 19,46. A formulação mais aceita pelos julgadores foi com $6,00 \%$ de farinha de uva, na temperatura de $140{ }^{\circ} \mathrm{C}$. Quanto a textura, os cookies não ficaram duros, quebradiços, adesivos ou gomosos, e ao avaliar os componentes principais, foi possível constatar a relação entre as análises físicas, tecnológicas e sensoriais.

Na elaboração dos cookies do estudo realizado por Tonon, (2019), foram utilizados farinha de trigo integral adicionados de farinha de uva e óleo de semente de uva, e realizado a avaliação de sensorial. Foram elaboradas 3 formulações diferentes, que substituíram a farinha de trigo integral por farinha de uva nas porcentagens de 15,10 e $5 \%$. Foi aplicada análise sensorial com 71 provadores não treinados a fim de verificar a aceitabilidade das amostras utilizando escala hedônica e sua intenção de compra. Os dados foram tratados através da análise de variância (ANOVA) e a comparação de médias foi feita utilizando teste de Tukey com 5\% de significância. Os dados obtidos indicaram que as formulações desenvolvidas foram 
aceitas em todos os atributos avaliados, assim a aplicação dos produtos obtidos do bagaço de uva é viável em produtos de panificação.

No entanto, De Melo et al. (2010), desenvolveu uma formulação de cookie, substituindo-se $25 \%$ da farinha de trigo por farinha de semente de uva, onde os cookies foram avaliados quando à composição centesimal e aceitabilidade por provadores não treinados e aleatórios $(\mathrm{n}=90)$. Os cookies com farinha de semente de uva apresentaram, em 100g, cerca de 447,18 calorias, $63 \%$ de carboidratos, $5 \%$ de proteína, $19 \%$ de lipídios e 5,7\% de fibras. Na aceitação global, o cookie de uva apresentou média de 8,03, correspondendo a "gostei muito" na escala hedônica. A substituição parcial da farinha de trigo por farinha de semente de uva proporcionou o desenvolvimento de um produto novo, com boa aceitabilidade sensorial e rica em fibras.

Em outro estudo realizado por Bennemann, et al., (2016) ao elaborar e utilizar uma farinha do bagaço de uva obtida a partir do subproduto gerado no processamento do suco de uva de uma agroindústria para a produção de muffins, observou que o incremento da farinha do bagaço de uva nas misturas, aumentou os teores de lipídeos, acidez, energia, fibra bruta e reduziu o teor de proteínas.

Outros estudos foram realizados com o intuito de analisar as diversas preparações utilizando a farinha de resíduo de uva. Nakov et al. (2020), desenvolveu um bolo, onde foram analisadas a composição química, propriedades nutricionais e características físicas deste bolo desenvolvido substituindo a farinha de trigo para pães por bagaço de uva em pó nas concentrações de 4\%, 6\%, $8 \%$ e 10\%. A adição de quantidades crescentes de pó de bagaço de uva aumentou gradualmente as cinzas, lipídios, proteínas, fibras, compostos fenólicos livres, antocianinas e teor total de polifenóis, bem como capacidade antioxidante (DPPH, FRAP), enquanto diminuição da umidade e pH. Os principais fenólicos fornecidos pelo bagaço da uva foram catequina, ácido gálico, quercitina, ácido protocatecuico, caempferol e apigenina. O conteúdo de ácidos fenólicos e flavonoides aumentou de 4,1 $\mathrm{mg} / \mathrm{kg}$ de MS (controle) para 26,4-60,9mg / $\mathrm{kg}$ de MS (bolo com bagaço de uva em pó 4\% $10 \%$ ). As coordenadas de cor $\mathrm{L} * \mathrm{e}$ a $*$ diminuíram, enquanto $\mathrm{b}$ *aumentada. O bolo contendo $4 \%$ de bagaço de uva em pó apresentou a melhor qualidade sensorial. Em outro estudo realizado por Huerta, et al. (2018), na elaboração de bolos, foram utilizados pré-misturas com diferentes concentrações de farinha do bagaço de uva (FBU) (15, 30 e 45\%), além da pré-mistura controle (0\%), avaliadas sensorialmente, como a pré-mistura contendo 45\% de FBU apresentou os melhores resultados para os atributos sensoriais, seguiu para as análises físico-químicas em conjunto a pré-mistura controle (PMC). No entanto, a prémistura selecionada (PMS) apresentou maiores teores de cinzas, lipídeos e fibra alimentar total em relação à PMC, embora amostras da PMS tenham apresentado menor volume e maior firmeza em relação aos bolos preparados a partir da PMC. Todas as amostras apresentaram estabilidade microbiológica. Quanto ao conteúdo de compostos bioativos e atividade antioxidante, as amostras que foram enriquecidas com FBU apresentaram teores consideráveis e maiores do que a amostra controle. Silva, R. Et al. (2020), também realizou um estudo através da elaboração de bolos com concentrações diferenciadas do bagaço de uva Cabernet Sauvignon (Vitis vinífera L.), sendo as amostras de $0 \%, 10 \%, 20 \%$ e $30 \%$, as quais foram avaliadas sensorialmente por 50 provadores não treinados, de ambos os sexos, maiores de 18 anos. A preparação com $10 \%$ de farinha proveniente do bagaço de uva obteve $88 \%(n=44)$ de aceitação na avaliação global e maior índice de aceitação entre as amostras, revelando que $86 \%(\mathrm{n}=43)$ certamente ou provavelmente comprariam o produto, sendo a mesma submetida às análises físico-químicas. Houve redução em $40 \%$ do teor de gordura quando comparado ao bolo tradicional.

Em estudo realizado por De Assis et al., (2018), onde foi desenvolvido um tipo de sorvete com adição de farinha obtida do bagaço de uva em substituição total a corantes e essências com o objetivo de avaliar a sua aceitabilidade e a composição nutricional da farinha. As concentrações utilizadas foram de 15,20 e $25 \%$ de farinha de bagaço de uva. As amostras foram submetidas ao teste de aceitação e intenção de compra, avaliadas por 50 provadores não treinados. Os resultados demonstraram que o sorvete com $15 \%$ de farinha de bagaço de uva em sua composição apresentou maior aceitação, 
entretanto, a formulação com $25 \%$ da farinha apresentou valores maiores quanto à intenção de compra. No referido trabalho, a farinha de bagaço de uva apresentou alto teor de fibras $(21,60 \mathrm{~g} / 100 \mathrm{~g})$, carboidratos e proteínas, e baixo teor de lipídeos, estando de acordo com os critérios propostos pela legislação da ANVISA para alimentos ricos em fibras. Portanto, o produto pode ser considerado promissor para a indústria de alimentos, apresentando características nutricionais desejáveis com baixo custo.

Bender, et al. (2016) elaborou outro produto utilizando o bagaço de uva do cultivar Marselan (Vitis vinífera) proveniente do processo de vinificação, para a elaboração de um snack com a farinha de casca de uva (FCU). Na formulação de snacks extrusados foram utilizadas concentrações de $9 \%$ e 18\%, em substituição à farinha de milho, representando $5 \%$ e $10 \%$ de fibra, respectivamente. Fibra $(58,01 \%)$, carboidratos $(17,62 \%)$ e cinzas $(12,46 \%)$ foram os principais constituintes da farinha de casca de uva, a qual teve pH de 3,51. Resveratrol (6,14mg. g-1), luteolina $(5,16 \mathrm{mg}$. g-1) e kaempferol (3,01mg. g1) foram os compostos fenólicos detectados em maior quantidade na FCU, indicando presença de antioxidantes na farinha. A formulação de snack contendo 9\% (5\% fibra) de FCU apresentou melhores resultados de aceitação com relação aos atributos cor, aroma e textura, comparada à formulação de snack padrão.

O bagaço de uva também tem sido avaliado quanto a sua possibilidade de aplicação como geleia. Em estudo realizado por Daniela Rocha et. al., (2020), foi produzido uma geleia de chia e bagaço da uva proveniente da Fazenda Santa Maria, situada na região do Vale do Médio São Francisco. Foram elaboradas três formulações de geleias 0\% (controle), 70\% e 90\% de concentração de bagaço de uva, as geleias foram avaliadas por 50 provadores não treinados, e os resultados obtidos da análise sensorial, revelaram que a amostra de geleia contendo 70\% de bagaço obteve boa aceitação, já intenção de compra de 16\% (n= 8) para certamente compraria e $60 \%(n=30)$ provavelmente compraria. Esta amostra obteve para os atributos textura, sabor aroma e impressão global 48\% (n=24), 50\% (n=25) e 56\% (n=28) para gostei muito, respectivamente, já intenção de compra de $16 \%(\mathrm{n}=8)$ para certamente compraria e $60 \%(\mathrm{n}=30)$ provavelmente compraria. Dessa maneira, os resultados obtidos abrem novas perspectivas para aproveitamento do bagaço da uva assim como para obtenção de um produto funcional, visto que as características sensoriais avaliadas foram bem aceitas pelos entrevistados.

Um estudo realizado por Teles (2014) com o intuito de avaliar o efeito do tempo e da temperatura de secagem sobre o teor de compostos fenólicos totais e a capacidade antioxidante do bagaço de uva para posterior obtenção de uma farinha e sua utilização na formulação de barras de cereais. Os experimentos foram conduzidos em diferentes temperaturas $\left(40^{\circ} \mathrm{C}, 50^{\circ} \mathrm{C}\right.$ e $60^{\circ} \mathrm{C}$ ) e os dados experimentais foram ajustados a seis modelos matemáticos que foram comparados frente aos melhores valores de coeficiente de determinação (R2) e da soma dos quadrados do resíduo (SQR).

Paralelamente, foi avaliado o comportamento dos compostos bioativos durante o processo de secagem, de acordo com o tempo e a temperatura ao qual foram expostos. O modelo de Page foi selecionado como o mais adequado para representar a secagem convectiva do bagaço de uva. Os tratamentos apresentaram diferença significativa em relação à retenção dos compostos fenólicos totais e capacidade antioxidante.

O bagaço seco a $60^{\circ} \mathrm{C}$ apresentou os maiores valores para fenólicos totais e capacidade antioxidante, sugerindo que esta temperatura foi capaz de inativar enzimas que catalisam a degradação dos compostos fenólicos. Foram realizados novos testes de secagem e a partir destes, foram obtidas a farinha do bagaço de uva que, após ser caracterizado, foi utilizado como ingrediente em diferentes concentrações para a formulação de barras de cereais. Esses produtos foram avaliados frente ao conteúdo de compostos bioativos e capacidade antioxidante. Ao finalizar o estudo foi constatado que as barras de cereais com os maiores teores de farinha apresentaram as maiores concentrações de compostos bioativos e capacidade antioxidante, sendo uma excelente alternativa para enriquecimento dos produtos alimentícios. 


\section{Considerações Finais}

Os estudos analisados (elaboração de produtos funcionais com uso do resíduo de uvas) tornam evidente a viabilidade e aplicabilidade da utilização do bagaço de uva, assim como dos subprodutos provenientes do seu processamento. Desse modo, pelos $75 \%$ dos estudos mostraram melhor aplicação no setor de farinhas, a segunda maior aplicação foi no de barras de cereais, com $15 \%$ dos estudos e a terceira aplicação foi no de geleias, com $10 \%$ dos estudos.

$\mathrm{Na}$ maioria dos estudos foram explorados os resíduos provenientes de vitivinícolas, e retrataram o potencial de conferir benefícios à saúde, no desenvolvimento de produto funcionais, reduzindo a quantidade destes resíduos na indústria e com possibilidade de redução de custos na produção destes produtos e benefício ao meio ambiente.

Estes achados são importantes sob o ponto de vista de saúde, pois propiciam a prevenção e promoção da saúde, como também podem atuar como modificadores de realidades econômicas, pois permitem agregar valor ao que seria expurgado e assim gerar renda para diversas famílias, o que por sua vez poderá mudar seu contexto social.

Como sugestão para trabalhos futuros, indica-se a aplicação e utilização do resíduo para outros fins, visto que o mesmo apresenta compostos e antioxidantes benéficos à saúde.

\section{Referências}

Abe, L. T. et al. (2007). Compostos fenólicos e capacidade antioxidante de cultivares de uvas Vitis labrusca L. e Vitis vinifera L. Food Science and Technology, 27, 394-400.

Abrafrutas (Associação Brasileira dos Produtores Exportadores de Frutas e Derivados (Abrafrutas), (2020). Dados estatísticos. Disponível em: https://abrafrutas.org/dados-estatisticos/. Acesso em 19/11/2020.

Abreu, J. P. de, (2018). Efeitos da adição de farinha de casca de uva orgânica (Vitis labrusca) sobre as características físicas, químicas e sensoriais no desenvolvimento de biscoito tipo cookie com alegação funcional. Dissertação de Mestrado.

Acan, B. G., Kilicli, M., Bursa, K., Toker, O. S., Palabiyik, I., Gulcu, M., Konar, N. (2021). Effect of grape pomace usage in chocolate spread formulation on textural, rheological and digestibility properties. LWT, 138, 110451. 10.1016/j.1wt.2020.110451.

Almeida, R. R. et al. (2019). Efeito da radiação ultravioleta C na qualidade e armazenamento pós-colheita de pequi. Nativa, 7, (1), 29-36.

Ângelo, P. M., \& Jorge, N. (2007). Compostos fenólicos em alimentos: uma breve revisão, Phenolic compounds in foods: a brief review. Revista do Instituto Adolfo Lutz, 66, (1), 1-9.

Anjos, J. R. C. dos. (2019). Características sensoriais de barras proteicas a base de clara de ovo enriquecidas com farinha de casca de uva (Vítis vinífera).

Araújo neto, J. R. de. (2019). Manejo de videiras para produção de uvas de mesa no Vale do São Francisco-Fazenda Nova Neruda. Trabalho de Conclusão de Curso. Brasil.

Barcia, M. T. (2014). Estudo dos compostos fenólicos e capacidade antioxidante de subprodutos do processo de vinificação. Tese (Doutorado em Ciência de Alimentos), Universidade Estadual de Campinas. p. 69.

Barreto, N. D. S. (2011). Qualidade, compostos bioativos e capacidade antioxidante de frutos de híbridos comerciais de meloeiro cultivados no CE e RN. 2011. 185f. Tese (Doutorado em Fitotecnia) - Universidade Federal Rural do Semiárido, Mossoró.

Bender, A. B. B. et al. (2016). Obtenção e caracterização de farinha de casca de uva e sua utilização em snack extrusado. Brazilian Journal of Food Technology, 19.

Bennemann, G. D. et al. (2016). Desenvolvimento e aceitabilidade de muffins adicionados de farinha de casca de uva das cultivares Ancelotta e Bordô. Revista da Universidade Vale do Rio Verde, 14 (2), 864-874.

Berry, C. (2002). Functional Foods. Quarterly journal of medicine, 95, 639-40.

Brasil. Agência Nacional de Vigilância Sanitária (ANVISA). (2003). Resolução RDC no 360, de 23 de dezembro de 2003 . Regulamento Técnico sobre Rotulagem Nutricional de Alimentos Embalados, tornando obrigatória a rotulagem nutricional. Diário Oficial da União. Brasília, DF.

Buttriss, J. L., \& Stokes, C. S. (2008). Dietary fibre and health: an overview. Nutrition Bulletin, 33, 186-200.

Caldas, T. W., Mazza, K. E. L., Teles, A. S. C., Mattos, G. N., Brigida, A. I. S., Conte-junior, C. A., Borguini, R. G., Godoy, R. L. O., Cabral, L. M. C., \& Tonon, R. V. (2018). Phenolic compounds recovery from grape skin using conventional and non-conventional extraction methods. Industrial Crops and Products, v. 111, 86-91.

Costa, A. C. S. (2012). Qualidade e atividade antioxidante na porção comestível e resíduos do processamento de acerola produzida no submédio do Vale do São Francisco. 2012. 116f. Dissertação (Mestrado em Agronomia) - Universidade Federal da Paraíba, Areia. 
Da Silveira, Márcia Adriana Gomes et al. (2020). A sustentabilidade do destino do bagaço da vinificação no Brasil. Research, Society and Development, 9(9), e247997197-e247997197.

Dani, C., Oliboni, L. S., Umezu, F. M., Pasquali, M. A. B., Salvador, M., Moreira, J. C. F., \& Henriques, J. A. P. (2007). Phenolic content and antioxidant activities of white and purple juices manufactured with organically or conventionally-produced grapes. Food and Chemistry Toxicology, 45, (12), 2574-2580.

De Assis, C. F. et al. (2018). Elaboração de sorvete à base de farinha de bagaço de uva e avaliação da sua aceitação. Revista Brasileira de Produtos Agroindustriais, 20(1), 33-38 2018.

De Mello, L. M. R. (2018). Panorama da produção de uvas no Brasil. Embrapa Uva e Vinho-Nota Técnica/Nota Científica (ALICE).

De Melo, V. S. et al. (2019). Aproveitamento da farinha de semente de uva na elaboração de biscoitos tipo cookie: composição química e aceitabilidade. BIOdiversidade: Educação, Saúde e Conservação, p. 56.

De Souza B., L. et al. (2014). Estudo da secagem de frutos tropicais do Nordeste. Revista Verde de Agroecologia e Desenvolvimento Sustentável, 9(1), 186190.

De Souza L., É. L., Moutinho, L. M. G., \& Campos, Luís H. R. (2016). Arranjo Produtivo Local da Fruticultura Irrigada do Vale do Submédio do São Francisco, Pernambuco/Bahia: Fluxos Comerciais e Dinamismo Local. Revista em Agronegócio e Meio Ambiente, 9(2), $273-303$.

Fagnani, R., \& Da Silva B., P. M. (2020). Formulação de iogurte concentrado enriquecido com farinha de semente de uva: atividade antioxidante e cinética de fermentação. Ensaios e Ciência C Biológicas Agrárias e da Saúde, 24, (2), 189-193.

Fao. (2021). Desperdício de alimentos tem consequências no clima, na água, na terra e na biodiversidade. http://www.fao.org.br/daccatb.asp.

Ferreira, M. D. (2017). Redução nas perdas pós-colheita em frutas e hortaliças. Um grande desafio. JC na Escola Ciência, Tecnologia e Sociedade: Mobilizar o conhecimento para alimentar o Brasil, p. 38.

Giuntini, E. B., Lajolo, F. M., De Menezes, E. W. (2003). Dietary fiber potential in IberianAmerican countries: Food, products and residues. Archivos Latinoamericanos de Nutrición, 53, (1), 14-20.

Gray, J. (2006). Dietary fibre - Definition, analysis, physiology and health. ILSI Europe Consise Monograph Series. Brussels, Belgium, ILSI Europe.

Henry, C. J. (2010). Functional foods. European Journal of Clinical Nutrition, 64, (7), 657-659.

Howlett, J. F., Betteridge, V. A., Champ, M. et al. (2010). The definition of dietary fiber - discussions at the Ninth Vahouny Fiber Symposium: building scientific agreement. Food \& Nutrition Research, 54.

Huerta, M. (2018). Bagaço de uva: aproveitamento, avaliação e aplicação em pré-mistura para bolo. Dissertação (Mestrado). Universidade Federal de Santa Maria, Centro de ciências rurais, p 58.

Machado, A. V., De Souza, J. A., Da Silva Novaes, R. (2015). Estudo cinético da secagem da uva Isabel para produção de uva passa. Revista Verde de Agroecologia e Desenvolvimento Sustentável, v. 10, (1), 7.

Mello, L. M. R. de. (2018). Relatório da avaliação de impactos econômicos das novas cultivares de uvas sem sementes BRS Vitória e BRS Isis no vale São Francisco - 2017. Centro nacional de pesquisa de uva e vinho, Bento Gonçalves: EMBRAPA uva $e$ vinho. https://bs.sede.embrapa.br/2016/relatorios/uvaevinho_2016_uvasemsemente.pdf.

Mello, L. M. R. de. (2017). Vitivinicultura brasileira: panorama 2017. Bento Gonçalves, RS: Embrapa Uva e vinho, 2018. (Embrapa Uva e Vinho. Comunicado Técnico, 207) Ação gerencial: Observatório Vitivinícola. http://ainfo.cnptia.embrapa.br/digital/bitstream/item/187913/1/Comunicado-Tecnico207. pdf

Nakov, G. Brandolini A, Hidalgo A, Ivanova N, Stamatovska V, \& Dimov I. (2020). Effect of grape pomace powder addition on chemical, nutritional and technological properties of cakes. LWT Elsevier BV, 134:109950. http://dx.doi.org/10.1016/j.lwt.2020.109950.

Natividade, M. M. P. (2010). Desenvolvimento, caracterização e aplicação tecnológica de farinhas elaboradas com resíduos da produção de suco de uva. 2010, 202f. Dissertação (Mestrado em Ciência dos Alimentos) - Universidade Federal de Lavras.

Perin, E. C., \& Schott, I. B. (2011). Utilização de farinha extraída de resíduos de uva na elaboração de biscoito tipo cookie. Trabalho de Conclusão de Curso. Universidade Tecnológica Federal do Paraná.

Pinheiro, E. S. (2008). Avaliação dos aspectos sensoriais, físico-químicos e minerais do suco de uva da variedade Benitaka (Vitis vinífera F.). 106f. Dissertação (Mestrado em Tecnologia de Alimentos) - Universidade Federal do Ceará, Fortaleza, 2008.

Poiani, M. R., \& Montanuci, F. D. (2019). Caracterizações físicas e tecnológicas e perfil de textura de cookies de farinha de uva e linhaça. Brazilian Journal of Food Technology, v. 22.

Ramos, R. V. R. et al. (2020). Sustentabilidade: utilização de vegetais na forma integral ou de partes alimentícias não convencionais para elaboração de farinhas. DEMETRA: Alimentação, Nutrição \& Saúde, 15, 42765.

Rufino, M. S. M., Alves, R. E. (2007). Metodologia Científica: Determinação de polifenóis extraíveis totais. Comunicado Técnico. Embrapa, Fortaleza.

Saad, S.M.I. (2006). Probióticos e prebióticos: o estado da arte. Revista Brasileira de Ciências Farmacêuticas, 42 , 1-16.

Scola, G. (2014). Atividade biológica de resíduos de vinificação de vitis labrusca. Tese (Doutorado) - Universidade de Caxias do Sul, Programa de PósGraduação em Biotecnologia. 
Siebeneichler, T. J. et al. (2016). Elaboração de pão com incorporação de farinha de uva. In: VI Salão Integrado de Ensino, Pesquisa e Extensão \& IIa Jornada de Pós-graduação da UERGS (ISSN: 2448-0010).

Silva, D. R., Quadros, C. P., \& Silva, C. De S. (2020). Bolo light enriquecido com farinha de bagaço de uva proveniente de produção vinícola. Brazilian Journal of Development, 6, (12), 96163-96171.

Silva, F. A. (2020). Desenvolvimento de iogurte caprino probiótico adicionado de derivados de uva isabel: propriedades nutricionais, tecnológicas e de funcionalidade. Tese (Doutorado em Nutrição) - Universidade Federal de Pernambuco, Recife.

Silva, J. A. da. (2018). Identificação de compostos fenólicos, macroantioxidantes e avaliação da atividade antioxidante do bagaço de uva proveniente da indústria de sucos no Vale do São Francisco. Tese de Doutorado.

Silva, L. M. R., Figueiredo, E. A. T., Ricardo, N. M. P. S., Vieira, I. G. P., Figueiredo, R. W., Brasil, I. M., \& Gomes, C. L. (2014). Quantification of bioactive compounds in pulps and by-products of tropical fruits from Brazil. Food Chemistry, 143, 398-404.

Siró, I., kápolna, E., kápolna, B., \& Lu-gasi, A. (2008). Functional food. Product development, marketing and consumer acceptance - A review. Appetite, 51, 456- 467.

Souza, A. V. de et al. (2012). Avaliação de antioxidantes em casca de Vitis sp. Revista Alimentus, 27-35.

Souza, J. C. (2008). Atividade antioxidante in vitro e in vivo de suco de uva e da norbixina. 2008. 94f. Dissertação (Mestrado em Alimentos e Nutrição) Universidade Estadual de Campinas, Campinas.

Souza, V. B. (2013). Aproveitamento dos subprodutos de vinificação da uva Bordô (Vitis labrusca) para obtenção de pigmentos com propriedades funcionais. Tese de Doutorado. Universidade de São Paulo.

Storck, C. R. et al. (2013). Folhas, talos, cascas e sementes de vegetais: composição nutricional, aproveitamento na alimentação e análise sensorial de preparações. Ciência Rural, v. 43, 537-543.

Tarcea, M., Rus, V., \& Zita, F. (2017). Insight of dietary fibers consumption and obesity prevention. Journal of Obesity \& Eating Disorders, 3, (1), 1-3.

Teles, A. S. C. et al. (2014). Estudo da secagem do bagaço de uva visando à sua utilização como ingrediente na formulação de barras de cereais. 66 f. Dissertação (Mestrado em Ciência e Tecnologia de Alimentos) - Instituto de Tecnologia, Departamento de Tecnologia de Alimentos, Universidade Federal Rural do Rio de Janeiro, Seropédica - RJ.

Tonon, G. C. (2019). Desenvolvimento e avaliação sensorial de cookie integral adicionado de farinha de uva e óleo de semente de uva. Trabalho de Conclusão de Curso. Universidade Tecnológica Federal do Paraná.

Tseng, A., \& Zhao, Y. (2013). Wine grape pomace as antioxidant dietary fibre for enhancing nutritional value and improving storability of yogurt and salad dressing. Food Chemistry, v.138, (1), 356- 365.

Tungland, B. C., \& Mayer, D. (2002). Nondigestible oligo - and polysaccharides (dietary fiber): their physiology and role in human health and food. Comprehensive Reviews in Food Science and Food Safety, 3, 73-92.

Vanderlei, D. R., De Quadros, C. P., \& De Sá, C. S. (2020). Geleia de bagaço de uva e chia proveniente da produção vinícola da região do submédio São Francisco. Brazilian Journal of Development, v. 6, (1), 4237-4248.

Vedana, M. I. S., Ziemer, C., Miguel, O. G., Portella, A. C., \& Candido, L. M. B. (2008). Efeito do processamento na atividade antioxidante de uva. Alimentos e Nutrição, v.19, (2), 159-165.

Williamson, C. (2009). Functional foods: what are the benefits? British Journal Community Nursing, 14, (6), $230-236$.

Xia, E., Deng, G. F., Guo, Y. J., \& Li, H. B. (2010). Biological activities of polyphenols from grapes. International Journal of Molecular Sciences, 11, (2), $622-646$. 